\title{
ELECTRON ANGULAR CORRELATION IN NEUTRINOLESS DOUBLE BETA DECAY AND NEW PHYSICS
}

\author{
A. $\operatorname{Ali}^{a}$ \\ Deutsches Elektronen-Synchrotron, DESY, 22607 Hamburg, Germany \\ A.V. Borisov ${ }^{b}$, D.V. Zhuridov ${ }^{c}$ \\ Faculty of Physics, Moscow State University, 119991 Moscow, Russia \\ Abstract.The angular correlation of the electrons in the neutrinoless double beta \\ decay $(0 \nu 2 \beta)$ is calculated taking into account the nucleon recoil, the $S$ and $P$ - \\ waves for the electrons and the electron mass using a general Lorentz invariant \\ effective Lagrangian. We show that the angular coefficient is essentially indepen- \\ dent of the nuclear matrix element models. We work out the angular coefficient in \\ several scenarios for new physics, in particular, in the left-right symmetric models.
}

\section{Introduction}

It is now established that the observed neutrinos have tiny masses and they mix with each other [1]. Theoretically, it is largely anticipated that the neutrinos are Majorana particles. Experimental evidence for $0 \nu 2 \beta$ decay would deliver a conclusive confirmation of the Majorana nature of neutrinos, establishing the existence of physics beyond the SM [2]. An extended version of the SM could contain tiny nonrenormalizable terms that violate lepton number (LN) and allow the $0 \nu 2 \beta$ decay. Probable mechanisms of LN violation may include exchanges by: Majorana neutrinos $\nu_{M} \mathrm{~S}[3,4]$ (the preferred mechanism after the observation of neutrino oscillations [1]), SUSY Majorana particles [5,6], scalar bilinears (SBs) [7], e.g. doubly charged dileptons (the component of the $S U(2)_{L}$ triplet Higgs etc.), leptoquarks (LQs) [8], right-handed $W_{R}$ bosons [4,9] etc. From these particles light $\nu$ s are much lighter than the electron and others are much heavier than the proton that gives two possible classes of mechanisms for the $0 \nu 2 \beta$ decay: long range (with the light $\nu$ s in the intermediate state) and short range mechanism. Our aim was to examine the possibility to discriminate among the various possible mechanisms contributing to the $0 \nu 2 \beta$-decays and the various sources of LN violation using the information on the angular correlation of the final electrons. We published a preliminary study along these lines in Ref. [10] and a more detailed study in Ref. [11]. Here, we summarize the main results of Ref. [11].

\section{Angular correlation for the long range mechanism of $0 \nu 2 \beta$ decay}

For the decay mediated by light $\nu_{M} \mathrm{~s}$, the most general effective Lagrangian is the Lorentz invariant combination of the leptonic $j_{\alpha}$ and the hadronic $J_{\alpha}$

\footnotetext{
${ }^{a}$ e-mail: ahmed.ali@desy.de

${ }^{b}$ e-mail: borisov@phys.msu.ru

${ }^{c}$ e-mail: dmitry.zhuridov@uwr.edu.pl
} 
currents of definite tensor structure and chirality $[12,13]$

$$
\mathcal{L}=\frac{G_{F} V_{u d}}{\sqrt{2}}\left[\left(U_{e i}+\epsilon_{V-A, i}^{V-A}\right) j_{V-A}^{\mu i} J_{V-A, \mu}^{+}+\sum_{\alpha, \beta}^{\prime} \epsilon_{\alpha i}^{\beta} j_{\beta}^{i} J_{\alpha}^{+}+\text {h.c. }\right]
$$

where the hadronic and leptonic currents are defined as: $J_{\alpha}^{+}=\bar{u} O_{\alpha} d$ and $j_{\beta}^{i}=\bar{e} O_{\beta} \nu_{i}$; the leptonic currents contain neutrino mass eigenstates, and the index $i$ runs over the light eigenstates. Here and thereafter, a summation over the repeated indices is assumed; $\alpha, \beta=V \mp A, S \mp P, T_{L, R}\left(O_{T_{\rho}}=2 \sigma^{\mu \nu} P_{\rho}\right.$, $\sigma^{\mu \nu}=\frac{i}{2}\left[\gamma^{\mu}, \gamma^{\nu}\right], P_{\rho}=\left(1 \mp \gamma_{5}\right) / 2$ is the projector, $\left.\rho=L, R\right)$; the prime indicates the summation over all the Lorentz invariant contributions, except for $\alpha=\beta=V-A, U_{e i}$ is the PMNS mixing matrix. The coefficients $\epsilon_{\alpha i}^{\beta}$ encode new physics, parametrizing deviations of the Lagrangian from the standard $V-A$ current-current form and mixing of the non-SM neutrinos.

The nonzero $\epsilon_{\alpha}^{\beta}$ for the particular SM extensions are collected in Table 1.

Table 1 .

\begin{tabular}{|c|c|}
\hline Model & Nonzero $\epsilon \mathrm{S}$ \\
\hline with $W_{R} \mathrm{~S}$ & $\epsilon_{V \mp \mp A}^{V \mp A}$ \\
\hline RPV SUSY & $\epsilon_{S+P}^{S \mp P}, \epsilon_{V-A}^{V-A}, \epsilon_{T_{R}}^{T_{R}}$ \\
\hline with LQs & $\epsilon_{S \mp P}^{S+P}, \epsilon_{V \mp A}^{V+A}$ \\
\hline
\end{tabular}

We have calculated the leading order in the Fermi constant and the leading contribution of the parameters $\epsilon_{\alpha}^{\beta}$ using the approximation of the relativistic electrons and nonrelativistic nucleons. We take into account the $S_{1 / 2}$ and the $P_{1 / 2}$ waves for the outgoing electrons and include the finite de Broglie wave length correction for the $S_{1 / 2}$ wave. Taking into account the nucleon recoil terms including the terms due to the pseudoscalar form factor we obtain the differential width in $\cos \theta$ for the $0^{+}(A, Z) \rightarrow 0^{+}(A, Z+2) e^{-} e^{-}$transitions:

$$
\frac{d \Gamma}{d \cos \theta}=\frac{\ln 2}{2}\left|M_{G T}\right|^{2} \mathcal{A}(1-K \cos \theta)
$$

where $\theta$ is the angle between the electron momenta in the rest frame of the parent nucleus, $M_{G T}$ is the Gamow-Teller nuclear matrix element and the angular correlation coefficient is

$$
K=\mathcal{B} / \mathcal{A}, \quad-1<K<1
$$

Expressions for $\mathcal{A}$ and $\mathcal{B}$ are given in Ref. [11]. The analytic expressions associated with the coefficients $\epsilon_{V \mp A}^{V+A}$ confirm the results of Ref. [4], while the expressions associated with $\epsilon_{V \mp A}^{V-A}, \epsilon_{S \mp P}^{S \mp P}, \epsilon_{T_{L}, R}^{T_{L, R}}$ transcend the earlier work. 


\section{Analysis of the electron angular correlation}

Consider the case of zero effects of all the interactions beyond the SM extended by the $\nu_{M}$ s (i.e., all $\epsilon_{\alpha}^{\beta}=0$ ), which we call the "nonstandard" effects. The values of $K=K_{0} \equiv K\left(\epsilon_{\alpha}^{\beta}=0\right)$ for various decaying nuclei are given in Table 2 .

Table 2.

\begin{tabular}{|c|c|c|c|c|c|}
\hline & ${ }^{76} \mathrm{Ge}$ & ${ }^{82} \mathrm{Se}$ & ${ }^{100} \mathrm{Mo}$ & ${ }^{130} \mathrm{Te}$ & ${ }^{136} \mathrm{Xe}$ \\
\hline$K$ & 0.81 & 0.88 & 0.88 & 0.85 & 0.84 \\
\hline
\end{tabular}

We will concentrate on the case of ${ }^{76} \mathrm{Ge}$ nucleus in the following. Using Table 1 and taking into account the fact that $\left|\mu_{\alpha}^{\beta}\right|$ are suppressed in comparison with $\left|\epsilon_{\alpha}^{\beta}\right|$ by the factor $m_{i} / m_{e}$ (the chiral suppression), we find the coefficient $K$ and the set $\{\epsilon\}$ of nonzero $\epsilon_{\alpha}^{\beta}$ s that can change the $1-0.81 \cos \theta$ form of the correlation for the SM plus $\nu_{M} \mathrm{~s}$, see Table 3 (the lower three entries).

Table 3

\begin{tabular}{|c|c|c|}
\hline SM extension & $\{\epsilon\}$ & $K$ \\
\hline$\nu_{M}$ & - & 0.81 \\
\hline$\nu_{M}+\mathrm{RPV} \mathrm{SUSY}$ & $\epsilon_{S+P}^{S+P}, \epsilon_{T_{R}}^{T_{R}}$ & $-1<K<1$ \\
\hline$\nu_{M}+\mathrm{RC}$ & $\epsilon_{V \mp A}^{V+A}$ & $-1<K<1$ \\
\hline$\nu_{M}+\mathrm{SLQ}$ & $\epsilon_{S \mp P}^{S+P}$ & $-1<K<1$ \\
\hline
\end{tabular}

They correspond to the following extensions of the SM: $\nu_{M}$ s plus RPV SUSY [6], $\nu_{M S}$ plus right-handed currents (RC) (connected with right-handed $W$ bosons [4] or vector LQs [8]), and $\nu_{M}$ s plus scalar LQs [8]. Hence, $K$ can signal the presence of this new physics.

Let us now consider some particular cases for the parameter space. We will analyze only the terms with $\epsilon_{V \mp A}^{V \mp A}$ as the corresponding nuclear matrix elements have been worked out in the literature. We use various types of QRPA $[14,15]$.

In the case of $|\langle m\rangle|=0$ the current lower bound $T_{1 / 2}>1.6 \times 10^{25} \mathrm{yr}$ for the ${ }^{76}$ Ge nucleus [16] yields the upper bounds on the parameters $\left|\mu_{V \mp A}^{V-A}\right|,\left|\epsilon_{V \mp A}^{V+A}\right|$ that give bounds on the parameters of the particular models [11]. The fact that the dependence of $K$ on the nuclear matrix elements is much weaker than the uncertainty in $T_{1 / 2}$ from this source was illustrated in Ref. [11].

In the case of $|\langle m\rangle| \neq 0, \cos \psi_{i}=0$, where $i$ depends on $\alpha, \beta$, for $\epsilon_{V+A}^{V+A} \neq 0$

$$
|\mu|^{2}=(7.9+10 K) \times 10^{12} / T_{1 / 2}, \quad\left|\epsilon_{V+A}^{V+A}\right|^{2}=(5.1-6.3 K) \times 10^{12} / T_{1 / 2},
$$

with $T_{1 / 2}$ in years, and for $\epsilon_{V-A}^{V+A} \neq 0$

$$
|\mu|^{2}=(7.7+10 K) \times 10^{12} / T_{1 / 2}, \quad\left|\epsilon_{V-A}^{V+A}\right|^{2}=(1.9-2.4 K) \times 10^{8} / T_{1 / 2} .
$$

The correlations among $\left|\epsilon_{V \mp A}^{V+A}\right|, T_{1 / 2}, K$ were used in the analysis of left-right 

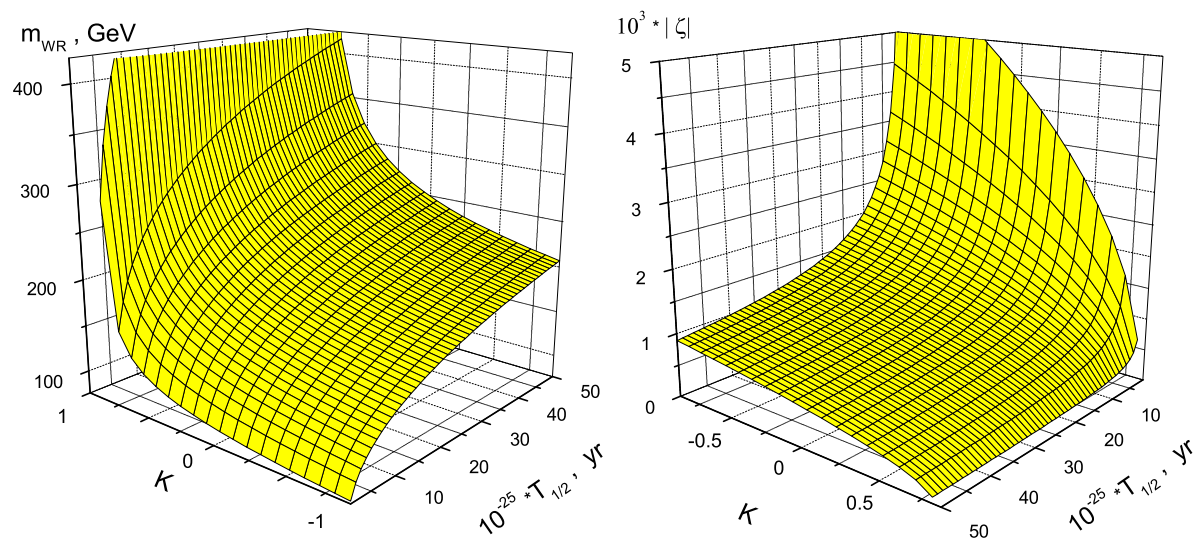

Figure 1: Correlation between the right-handed $W$-boson mass $m_{W_{R}}$ (left) or the mixing $\zeta$ (right), the angular coefficient, and the half-life $T_{1 / 2}$ for the $0 \nu 2 \beta$ decay of ${ }^{76} \mathrm{Ge}$.

symmetric models [17]. In the model $S U(2)_{L} \times S U(2)_{R} \times U(1)$ we have

$$
m_{W_{R}}=m_{W_{L}}\left(\epsilon /\left|\epsilon_{V+A}^{V+A}\right|\right)^{1 / 2}, \quad \zeta=-\arctan \left(\left|\epsilon_{V-A}^{V+A}\right| / \epsilon\right)
$$

for the mass of the right-handed $W$ boson and its mixing angle $\zeta$ with the left-handed one. The correlation among $m_{W_{R}}(\zeta), K$, and $T_{1 / 2}$ is shown in Fig. 1 left (right) for conservative value $\epsilon=10^{-6}$ for the mixing parameter $\epsilon=\left|U_{e i} V_{e i}\right|$. It is clear that the closer is $K$ to 1 for the fixed value of $T_{1 / 2}$ the stronger is the lower bound on $m_{W_{R}}$ (the upper bound on $\zeta$ ). We have shown also that the sensitivity of the angular correlation to the $W_{R}$ mass increases with decreasing values of the effective Majorana neutrino mass $|\langle m\rangle|[11]$.

\section{Acknowledgments}

We thank Alexander Barabash and Fedor Šimkovic for helpful discussions. One of us (DVZ) would like to thank DESY for the hospitality in Hamburg where a good part of this work was done.

\section{References}

[1] Particle Data Group: W.-M. Yao et al., J. Phys. G33, 1 (2006).

[2] S.R. Elliot and P. Vogel, Ann. Rev. Nucl. Part. Sci. 52, 115 (2002); P. Vogel, arXiv:hep-ph/0611243.

[3] Ya.B. Zel'dovich and M.Yu. Khlopov, JETP Lett. 34, 141 (1981); Sov. Phys. Usp. 24, 755 (1981); M.G. Shchepkin, Sov. Phys. Usp. 27, 555 (1984). 
[4] M. Doi, T. Kotani, and E. Takasugi, Prog. Theor. Phys. Suppl. 83, 1 (1985).

[5] R.N. Mohapatra, Phys. Rev. D34, 3457 (1986); J.D. Vergados, Phys. Lett. B184, 55 (1987); M. Hirsch, H.V. Klapdor-Kleingrothaus, and S.G. Kovalenko, Phys. Rev. Lett. 75, 17 (1995); Phys. Lett. B352, 1 (1995); Phys. Lett. B403, 291 (1997); Nucl. Phys. Proc. Suppl. B52, 257 (1997); Phys. Rev. D57, 1947 (1998); K.S. Babu and R.N. Mohapatra, Phys. Rev. Lett. 75, 2276 (1995); A. Faessler, S.G. Kovalenko, F. Simkovic, and J. Schwieger, Phys. Rev. Lett. 78, 183 (1997).

[6] M. Hirsch, H.V. Klapdor-Kleingrothaus, and S.G. Kovalenko, Phys. Lett. B372, 181 (1996); B381, 488 (Erratum) (1996); H. Päs, M. Hirsch, and H.V. Klapdor-Kleingrothaus, Phys.Lett. B459, 450 (1999).

[7] H.V. Klapdor-Kleingrothaus and U. Sarkar, Phys. Lett. B554, 45 (2003).

[8] M. Hirsch, H.V. Klapdor-Kleingrothaus, and S.G. Kovalenko, Phys. Rev. D54, 4207 (1996).

[9] M. Hirsch, H.V. Klapdor-Kleingrothaus, and O. Panella, Phys. Lett. B374, 7 (1996).

[10] A. Ali, A.V. Borisov, and D.V. Zhuridov, arXiv:hep-ph/0606072.

[11] A. Ali, A.V. Borisov, and D.V. Zhuridov, Phys. Rev. D 76, 093009 (2007).

[12] H. Päs, M. Hirsch, H.V. Klapdor-Kleingrothaus, and S.G. Kovalenko, Phys. Lett. B453, 194 (1999).

[13] G. Gamov and E. Teller, Phys. Rev. 49, 895 (1936); S.F. Novaes, in "Particle and Fields", Proc. 10th J.A. Swieca Summer School, São Paulo, Brazil, 31 Jan - 12 Feb 1999 (World Scientific, Singapore, 2000) [arXiv:hep-ph/0001283].

[14] G. Pantis, F. Šimkovic, J.D. Vergados, and A. Faessler, Phys. Rev. C53, 695 (1996).

[15] M. Kortelainen and J. Suhonen, Phys. Rev. C75, 051303 (2007).

[16] C.E. Aalseth et al., Phys. Rev. D70, 078302 (2004).

[17] J.C. Pati and A. Salam, Phys. Rev. D10, 275 (1974); R.N. Mohapatra and J.C. Pati, Phys. Rev. D11, 566, 2558 (1975); G. Senjanovic and R.N. Mohapatra, Phys. Rev. D12, 1502 (1975); R.N. Mohapatra and G. Senjanovic, Phys. Rev. D23, 165 (1981). 